\section{ESTUDIOS PIONEROS EN TORNO AL ORIGEN DEL LENGUAJE NATURAL}

\author{
Jorge Martínez Contreras \\ Departamento de Filosofía \\ Universidad Autónoma Metropolitana-Iztapalapa (México)
}

\section{PIONEER STUDIES ON THE ORIGIN OF NATURAL LANGUAGE}

\begin{abstract}
It has being an ancient desire to ask apes what their natural lives are. De la Mettrie was the first to propose, in the $18^{\text {th }}$ $C$. , that the sign language of deaf adults could be used with them since they do not speak. We enhance here some of the pioneering projects of the two strategies for this endeavor: the use of ASL and the utilization of lexigrams and computers. Besides the ancient communicative quest with them, the evolutionist's perspective has seen in these studies a way to find out how the natural language (NL) emerged in hominids. If it is clear that apes do no possess totally the $N L$, the linguistic turn in primatology has left way, as in philosophy, to more complex field and laboratory cognitive studies of less anthropocentric nature.
\end{abstract}

KEY WORDS: Natural language; simian language; lexigrams; Washoe; Premack; Rumbaugh.

\section{INTRODUCCIÓN}

Si los simios o póngidos son los seres vivos más cercanos a nuestro ancestro común ¿se puede pensar buscar en ellos el origen del lenguaje? ¿Es posible enseñarles a hablar por medio del lenguaje natural (LN)? ¿Cómo se han llevado a cabo varios de esos intentos? ¿Y, si hablaran, qué nos dirian?

Respondemos positivamente a la primera pregunta, pero sólo desarrollaremos las siguientes. Desde los estoicos (Martínez-Contreras, 2002a) hasta nuestros días muchos piensan que los animales "hablan", aunque no podamos entenderlos. Los dayaks de Borneo creían que los orangutanes hablaban, pero que callaban ante los humanos para que nos los pusieran a trabajar. Muchos pensadores han deseado buscar un medio de comunicación con los animales, en especial con los póngidos, a partir de su descubrimiento occidental en el siglo XVI (Martínez-Contreras, 2006). De la Mettrie, en el XVIII, propuso por primera
RESUMEN: Tratar de hablar con los simios y preguntarles cosas sobre su vida natural es un viejo deseo. De la Mettrie propone, en el s. XVIII, que se les enseñe el lenguaje de los sordos en vista de que no pueden hablar. Resaltamos aqui algunos estudios pioneros en relación con las dos estrategias llevadas a cabo para tratar de hablar con los simios: el uso del lenguaje americano de sordos (ASL) y el de los lexigramas y computadoras. Además del viejo interés interrogativo humano hacia ellos, la perspectiva evolucionista ha visto en estos estudios un medio de investigar cómo surgió el lenguaje natural (LN) en homínidos. Si queda claro que los simios no poseen totalmente el $L N$, el giro lingüistico en primatología ha cedido, como en filosofía, el paso a estudios cognitivos más complejos, de campo y de laboratorio, de orientación menos antropomórfica.

PALABRAS CLAVE: Lenguaje natural; lenguaje en simios; lexigramas; Washoe; Premack; Rumbaugh.

vez que se debiera tratar de enseñarles el lenguaje de los sordos. A pesar de los trabajos pioneros y seminales sobre la cognición en póngidos por Köhler y Yerkes en los años veinte y treinta del siglo XX (Martínez-Contreras, 2002b), habrá que esperar hasta la mitad de dicho siglo para ver avanzar semejantes intentos. En lo que sigue analizaremos algunos trabajos pioneros del último medio siglo para hablar con los simios.

Las controversias en relación con las capacidades lingüisticas de los primates no humanos son intensas, científica y metafísicamente hablando, a pesar de que se trataría sólo de cuestiones técnicas. Algunas de estas investigaciones científicas fueron: la Comunicación por medio del ASL (American Sign Language) (CASL) o por medio de lexigramas y ordenadores (CLO). Los más recientes estudios han potenciado ese intento método. El ASL es utilizado por una población numerosa de humanos, pero los lenguajes artificiales son puramente y simplemente inventados. 
Allen y Beatrix Gardner (Gardner \& Gardner, 1989a, 1989b), son los pioneros de CASL y sus investigaciones han adquirido un renombre internacional. Uno de sus discípulos, Roger Fouts (1979, 1989a, 1989b), retoma estas investigaciones y adopta una vía original al tratar de hacer que los chimpancés se comuniquen entre sí por ese medio: demuestra que pueden enseñar el ASL a otros chimpancés, sin un intermediario humano ${ }^{2}$. Posteriormente, David Premack (Premack \& Woodruff, 1978, Premack \& Premack, 1983), inaugura las investigaciones CLO: sus escritos sobre la chimpancé Sarah han alcanzado un renombre internacional. El equipo de Duane Rumbaugh, retomará la idea CLO, pero a diferencia de Premack, quien utiliza piezas de plástico magnetizadas sobre un tablero magnético, usará ordenadores, con asombrosos resultados. Más tarde, su esposa, E. Sue Savage-Rumbaugh, dará un paso decisivo al interesarse en los bonobos, cuya inteligencia sobrepasa a la de los chimpancés troglodytes, siguiendo en sus trabajos un método mucho más etológico que experimental. Veamos algunos de estos ejemplos particulares.

\section{El lenguaje ASL}

Los Gardner comienzan en junio de 1966, con Washoe ${ }^{3}$ (1965-2007), hembra de origen salvaje de entre 8 y 14 meses. Dos hipótesis fundamentales guían su trabajo:

a) Si el lenguaje se adquiere a través de actividades sociales particulares, ¿es la estimulación en un medio humano necesaria y suficiente para ello?

b) ¿Es el ASL adecuado para llevar a bien este proyecto?

Los Gardner llegan a la conclusión asombrosa y visionaria de que pueden realizar la CASL con los chimpancés. Piensan evitar el fracaso de Hayes ${ }^{4}$, quien no pudo comunicarse oralmente con su hembra Viki (sic). Si los chimpancés, carentes de una laringe adecuada, no pueden hablar, sí disponen de manos asombrosamente dúctiles y son muy inteligentes. Es así como el chimpancés se volvió "sordo" y aprendió el ASL, lenguaje manual que permite, además, un reforzamiento conductista. Hacer signos sería el equivalente de empujar una palanca en una caja de condicionamiento skinneriano. Los Gardner realizan dos subproyectos. El primero comienza con Washoe en 1966. La hembra será criada como un niño y no como un animal doméstico. A los 4,5 años, su vocabulario cuenta con 130 signos. El segundo subproyecto (1972) es con los chimpancés Moja, Tatu, Eli y Dar, nacidos en cautiverio. Todos son expuestos, igual que aquélla, al ASL a partir del cuarto dia de vida y viven en la casa de los Gardner con cierto grado de libertad. Los chimpancés hacen signos entre sí, aunque esto incluye necesariamente la participación de humanos. Se utilizan también las técnicas de condicionamiento que Fouts había ya empleado para entrenar a los primates en CASL. El primer signo hecho por un chimpancé es observado a los tres meses de edad; luego, a los dos, los chimpancés ya poseen más de 50 . Son capaces de hacer un uso abstracto del signo para decir "no" y utilizan frases múltiples combinando un nombre y una acción, una acción y una localización, pero también la posición, la denominación y la recurrencia. Una vez introducido el ASL, este lenguaje se revela a la vez robusto y autosuficiente en la comunidad simiesca.

El método de colecta de los datos es por medio de una videograbadora. Se trata de muestras "exhaustivas" de ciertos comportamientos de "signar", donde se señalan después los contextos de sus realizaciones. Además de estas pruebas con doble control, los Gardner buscarán siempre un acuerdo inter-individual, con el fin de reducir al máximo la subjetividad del observador. Para argumentar en favor de la fiabilidad de este procedimiento, los Gardner harán venir expertos del ASL "inocentes". Se demuestra así que Washoe utiliza verdaderamente los signos del ASL, y no aproximaciones más o menos fortuitas. Para ello, una regla draconiana instituida: para decidir si el chimpancé ha adquirido un signo es requerida una utilización espontánea y apropiada del signo durante 15 días seguidos, constatado por tres observadores independientes. Espontánea significa que los simios no han sido incitados de una manera u otra a utilizar el signo en cuestión. La primera ocurrencia del uso de un signo es generalmente inesperada y sorprendente. Los instructores tienen entonces tendencia a tratar a los signos ambiguos de Washoe como signos que le han sido enseñados anteriormente. Un fenómeno de refuerzo a estímulos puede entonces producirse dentro de las reglas del condicionamiento conductista clásico. En relación con las combinaciones de signos entre sí, los Gardner consideran que la tendencia de Washoe para producir los mismos ha sido no solamente espontánea sino también precoz, a partir de un repertorio de una decena de signos. Su producción se incrementa al mismo tiempo que su vocabulario, 
así como su dominio del ASL, lo que le permite combinar hasta cinco signos en la misma "frase". Una real comunicación en dos sentidos entre el hombre y el chimpancé se creó así. En ese sentido, nos acercamos a la posibilidad de preguntarles a los simios sobre su vida, como se requería desde hacía siglos.

En su segundo subproyecto, los Gardner recurrieron a "signos del día" y a "descripciones PCM" (place, configuration and movement). El lugar "P" (place), del signo sobre el cuerpo, la configuración de la mano (C) y su movimiento (M). Las descripciones PCM están destinadas a aumentar el rigor, no sólo en la obtención de signos, sino también en su descripción, gracias a lo que se ha llamado descripciones estándar del Diccionario de signos ASL. Este diccionario organiza la descripción de todos los signos utilizados en ASL a partir de tres parámetros PCM. Para los Gardner, se demuestra sin error posible que los chimpancés tienen capacidades lingüísticas interesantes y producen signos apropiados al contexto. Al final de los años 70, Washoe se une a un nuevo equipo formado en la Universidad de Oklahoma.

\section{LeXigramas y computadoras (EO)}

Fouts desarrolla un nuevo método para enseñar el lenguaje de signos a los simios, la modelación (modelling) para ver si los simios pueden educar a sus congéneres. Esta técnica consiste en modelar sobre las manos del primate los signos a efectuar, en vez de mostrárselos simplemente. Fouts pudo mostrar, en su tesis doctoral, que Washoe hacia incontestablemente menos errores con su técnica, inspirada en el quehacer humano. El único problema era el riesgo del contacto del investigador con un simio adulto, jmucho más peligroso que frente a cualquier sordo!

Fouts promueve también que los primates se comuniquen entre ellos por medio del ASL, fuera de toda presencia humana, y que se lo enseñen a otros chimpancés. Pero, ¿puede el hombre darle a los primates útiles simbólicos que les permitan comunicarse en conjunto?, ¿es obligatorio introducir un operador humano en el "diálogo" o podemos prescindir de él? y ¿con qué resultados?

En 1973 Booee y Bruno son los cobayas de esta primera tentativa. Su vocabulario es mantenido al principio arti- ficialmente muy bajo, alrededor de 40 signos solamente, para poder determinar la influencia de Washoe sobre ellos, pero este experimento no tiene mucho éxito. Cuando se encuentran solos, Booee y Bruno desprecian el ASL y prefieren métodos más naturales de comunicación. El comportamiento de Washoe y de Ally -otro simio "hablante" de la colonia- es sin embargo diferente y los dos monos se comunican a veces en ASL. En 1974, Ally es introducida en el grupo compuesto por Booee y Bruno; este último y Ally intercambian signos, principalmente en relación con la comida y el juego. Es sin duda ya un resultado apasionante y problemático, pero permanece insuficiente. En 1979 un pequeño chimpancé, Loulis, quien es adoptado, en una sesión bastante asombrosa, por Washoe (quien había perdido a dos hijos). Fouts nos explica:

La experiencia dura cinco meses. Loulis no interactúa solamente con Washoe, sino también con otros tres chimpancés hablantes (...). Los observadores reciben al principio consignas de silencio gestual. (...). Los investigadores no deben interactuar con Washoe más que en inglés oral, que ella comprende perfectamente. Han recibido igualmente instrucciones muy estrictas en relación con la obtención de los datos. El gesto, su contexto y los comportamientos no verbales que lo acompañan deben ser considerados. La orientación de Loulis durante la interacción debe ser indicada: ¿está orientada hacia alguien (hombre o simio) cuando hace un signo? ¿Mira a alguien de frente? Los instructores deben también (anotar en) un cuestionario (...) tres niveles de estado (de erección) piloso (Fouts, 1982: 160; nuestra traducción).

La enseñanza de los signos a Loulis fue más allá de la simple transmisión de la información. Washoe, con este hijo adoptivo, adoptó todos los métodos aprendidos: el modelaje, la demostración de signos sobre el cuerpo de Loulis, exactamente como lo hacen los padres humanos con los niños sordos. Loulis imita a veces los signos de Washoe, pero con cierto retraso. Más allá de esta enseñanza, Loulis y Washoe juegan juntos: ésta, por ejemplo, se cubre los ojos y trata de encontrar a aquélla. Al respecto, Fouts y sus colegas subrayan que jugaban ese mismo juego con Washoe diez años antes. Washoe y Loulis toman alternativamente la iniciativa del juego. Una relación recíproca que se ha instalado entre los dos primates. Washoe también le enseña a Loulis "buena educación". Le muestra y lo disciplina -cosa que nadie le había pedido- lo que 
es un "buen comportamiento" en cautiverio: Washoe, por ejemplo, que utilizaba usualmente un signo distinto al de sus compañeros para designar a un cobertor, rápidamente incluirá en su vocabulario un nuevo signo de cobertor, el usado por los otros chimpancés.

Con Premack, las investigaciones sobre las capacidades lingüisticas de los grandes simios van a tomar una dirección muy diferente: se pasará del signo manual al ideograma (CLO). Aquél construye su propio lenguaje artificial con ideogramas de plástico magnetizados que evitan el sesgo debido a la memorización en relación con la reproducción y la comprensión de los símbolos. En dos proyectos simétricos considera que se pueden aportar luces decisivas sobre la relación entre el lenguaje y los procesos cognitivos más generales. El primero, consistiría en privar a un niño de su ambiente lingüístico para estudiar las consecuencias negativas, a la manera de un niño salvaje; un programa éticamente imposible. El segundo consiste en enseñarles el LN por medio de una gramática general. Una de sus preguntas se centraba en la posibilidad de obtener una gramática en los dominios no lingüísticos, como el juego, la verificación de utensilios, el arte, etc.; pero fracasó. El lenguaje y el juego no son sistemas comparables. Para saber hablar, un organismo debe saber que es capaz de manipular un cierto número de elementos del lenguaje que incluyen palabras, frases, conceptos sencillos, como el color, la talla; además de la cópula, los cuantificadores, lo hipotético, etc. El póngido no está, sin duda, genéticamente programado para hablar, pero el hombre tampoco lo está para volar y sin embargo vuela por medio de ayudas tecnológicas. Por ello, por medio de la tecnología adecuada, se le pueden preguntar cosas sobre su vida. El experimento de Premack no deja tampoco de evocar el sueño de La Mettrie y de los ilustrados que querian conocer el universo mental de los póngidos.

En 1970, Premack se asocia con un lingüista, A. Schwartz, con el fin de comunicar con la chimpancé Sarah, esta vez por medio del joystick de una computadora. Los resultados son juzgados poco prometedores y el proyecto es detenido. Pero el lenguaje que inventó lo hará célebre. Éste se compone de objetos de plástico imantando y coloreado con formas barrocas que se pegan sobre un tablero. Cada una de las "palabras" representa un objeto según una correspondencia rigurosa y no icónica sino arbitraria, como los fonemas humanos. Las frases se construyen como en
Chino, verticalmente. No hay gramática, pero Sarah dispone de un diccionario: una gran caja en el interior de la cual se encuentra el vocabulario disponible. Sarah, como los humanos, pudiera olvidar los signos que menos se usan. El experimentador refuerza el comportamiento de la chimpancé. Por ejemplo, cuando expresa "manzana" es recompensada si aporta efectivamente una manzana. Se debe escribir enseguida "da manzana", y después "Sarah da manzana". Una pequeña pieza de plástico se vuelve entonces una palabra cuando las características que le son atribuidas son aquéllas del objeto designado. Premack estima que un cierto número de las capacidades cognitivas de la chimpancé han sido modificados por el entrenamiento lingüístico y simbólico que han recibido. Sarah, en 1983, comprende la proporción, la analogía, incrementa sus capacidades intencionales, representa las acciones, sus causas, así como sus consecuencias, lo mismo que la conversación de los objetos a través de la acción. ¿Pero es capaz de describir con palabras las acciones que efectúa? ¿Puede describir intercambios en los que participaba cuando aún no estaba educada? Además, adquiere un vocabulario extenso, aunque sea evaluado como un criterio estricto, como el que es utilizado por Premack. Un ejemplo simple puede ser dado. Las caracteristicas de la manzana son: roja/redonda con una cola, y Sarah muestra que ella ha señalado la "palabra" cuando le atribuyen todas estas características que ella ha atribuido al objeto en una experiencia precedente.

¿Cuáles son las otras capacidades que puede mostrar Sarah? En 1983, es capaz de responder a preguntas operativas que muestran claramente que puede manejar la pareja diferente/idéntico ¿Pero comprende el signo de interrogación? Comprende en efecto que es la marca que señala la ausencia de una información. Es interesante notar que es capaz de nombrar objetos e incluso de utilizar la palabra "nombre", por ejemplo, para conocer el nombre de un objeto nuevo. Distingue "y", así como "o", el orden de las cosas y expresa sus emociones a través del lenguaje de Premack. ¿Es la conexión entre la palabra y el lenguaje clara? Sarah combina visiblemente sus emociones con la manipulación de las palabras en plástico, que aplica muy a menudo sobre el tablero que ha sido previsto a este efecto, con una intensidad repetida.

Duane Rumbaugh (1977) retoma el trabajo de Premack. Él trabaja también sobre el aprendizaje de un lenguaje artifi- 
cial por parte de los chimpancés e inventa otro: el Yerkish ${ }^{6}$. Con ayuda de lingüistas elabora un lenguaje que será enseñado a los primates por medio de una computadora. De ahí, surgirán tres proyectos: LANA (LANguage, Analogue), el Animal Model Project (AMP) y el proyecto Kanzi.

El propósito esencial del proyecto LANA es probar las capacidades lingüísticas del chimpancé Lana por medio del Yerkish. Este lenguaje es ideográfico y usa lexigramas no icónicos, es decir, que no se parecen en nada a aquello a lo que hacen referencia, clasificados siguiendo una semántica primitiva. El chimpancé aprende primero a asociar un lexigrama con el objeto al que se refiere y luego logra hacer con ellos frases. Las pruebas con múltiple elección son bastante sencillas y le son propuestas al animal que recibe una recompensa cuando sus respuestas son correctas. A partir de ahí, los investigadores analizan "las conversaciones" del simio. El sistema LANA hace posible que este procedimiento sobre la capacidad de registrar sistemáticamente lexigramas sea controlada objetivamente por medio de un teclado manipulado desde una habitación separada. Cada tecla representa un lexigrama (una "palabra"), es decir, que sólo tiene una significación. Lana utiliza los conceptos "nombre de" y "relación"; conceptos numéricos y establece usos nuevos de frases al apoyar sucesivamente sobre varias teclas. El tamaño de su vocabulario alcanza 75 unidades. Al principio, Lana tiene acceso a frases ya totalmente hechas Ilamadas holofrásticas, que son globalmente disparadas cuando el chimpancé acciona una tecla que corresponde a sólo uno de sus elementos. Los investigadores del programa estiman encontrar la confirmación de que las fundaciones del lenguaje deben ser buscadas en una inteligencia más general que tiene su origen en la posibilidad de ordenar los objetos, de catalogarlos, de reunirlos con la realidad y de codificarlos simbólicamente. Los chimpancés tendrían entonces la posibilidad de hablar, pero no lo harían espontáneamente: procesos ocultos deben poder ser activados para permitirles hablar verdaderamente. Dicho de otra forma, no existiría, como sostienen los Chomskyanos, una especificidad genética del lenguaje. Tres perspectivas parecen prometedoras en este proyecto LANA: profundizar sobre las capacidades lingüísticas de los simios por medio de funciones todavía en desarrollo, por ejemplo el uso del tiempo; revisar la definición del lenguaje; tomar al chimpancé como modelo animal del lenguaje. Este último punto conduce directamente al segundo proyecto del equipo de la Universidad de Georgia, el Animal Model Project (AMP).
EI AMP quiere ser un instrumento para interrogarse sobre la naturaleza del lenguaje, pero también relanzar los estudios sobre comunicación inter-específica entre chimpancés y humanos. Se distingue de otros proyectos en la medida en que no trata de probar cuáles pueden ser los límites superiores del lenguaje de los póngidos. Un argumento en su favor es la existencia de la solicitud y de la denominación. En efecto, la indicación espontánea es la primera capacidad semántica que deben mostrar sin entrenamiento Sherman y Austin, los chimpancés cobayas del proyecto. Los investigadores, obviamente, no tienen un control a priori sobre lo que los chimpancés expresan en un momento dado. ¿Cómo determinar, entonces, si un chimpancé se comporta de manera indicativa?: un control es concebido para asegurarse que seleccionan verdaderamente los objetos que van a nombrar y que no se apoyan sobre las teclas del tablero de manera azarosa, que son capaces de una capacidad simbólica efectiva. La pareja Savage-Rumbaugh (1986, 1994), desarrolla una serie de experimentos ingeniosos, al disociar explícitamente el proceso de solicitud del proceso de denominación, y al asegurarse que el primate designa bien, sin verlos, los objetos sobre los cuales emplea signos. Las recompensas recibidas no tienen ninguna relación con lo que es designado y un dispositivo experimental solicita al experimentador comunicar con el simio, separados por una gruesa pared. El animal debe, en primer lugar, apoyar sobre la tecla que corresponde al objeto deseado; enseguida, lo debe ir a buscar sobre una mesa del otro lado del muro, para después Ilevarlo al instructor.

No tenemos espacio para analizar la segunda serie de experimentos que conlleva el estudio de la capacidad simbólica; digamos que Savage-Rumbaugh responderán positivamente a esta pregunta.

En conclusión, ¿han podido los psicólogos hablar con los simios? La respuesta, aquí otra vez, dependerá de cómo y con qué requisitos utilicemos la expresión "hablar".

La primera conclusión daría razón a los detractores de los intentos por establecer una comunicación idéntica con animales no humanos. No tendrían, ni siquiera los simios más cercanos a nosotros, las capacidades mentales, computacionales, para establecer una doble articulación como la tenemos los humanos. Por ello, añadirian, tampoco pueden tener creencias y pensar, en el sentido humano de la palabra. Se trata de una limitación importante, pues en 
vez de poseer una capacidad ilimitada de producir combinaciones, no llegan más allá de la expresión de unos cuatrocientos monemas (Martinet dixit) o "palabras", así como de combinaciones limitadas entre ellos. Ahora bien, hay humanos que viven en situación semejante: los niños en edad prelingüística y los niños salvajes, por sólo citar dos grupos: ¿se pudiera afirmar que un niño menor de dos años no piensa pues no posee todavía el lenguaje? Este tema invita, desgraciadamente, a afirmaciones de principio que no deseamos analizar aquí. Concedámosles que sólo los humanos gozamos del lenguaje natural.

Ahora bien, la distancia en expresión lingüística entre el póngido educado y cualquier otro mono educado, sin mencionar a otros mamíferos muy inteligentes como cánidos, delfines o paquidermos, es tal vez tan grande como la que existe entre los póngidos y nosotros. Si lo que les podemos preguntar no es tan sofisticado como lo que se pretendía en el siglo XVIII, es asombrosamente interesante, pues nos manifiestan gustos, deseos, rechazos, etc. Nos expresan que los objetos nuevos pueden ser clasificados, un poco a la manera de Hume, asociando hasta tres significados, como "redonda", "roja" y "con cola" para una manzana. En este sentido, los estudios han sido muy exitosos y anuncian, ahora que se trabaja con el más cercano a nosotros de los póngidos, el bonobo, resultados aún más prometedores.

¿Les hemos podido preguntar sobre su vida y qué han respondido? Con las limitaciones del lenguaje, debemos aceptar que los póngidos sí han respondido. Se interesan en cosas muy concretas, como la comida, o goces que llamariamos hedónicos, que varian de individuo en individuo. Se interesan también mucho en el sexo. Sin embargo, la mayoría de nosotros tampoco se plantea cotidianamente

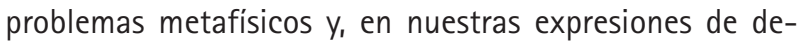
seos, los humanos también pensamos y hablamos principalmente de la comida, del hedonismo y del sexo. Lo que nos diferencia, aquí otra vez, es una situación de grado y no de naturaleza.
Por ello, no nos encontramos en medida de señalar cómo ha surgido el lenguaje natural humano a partir de formas animales más primitivas. Pero no dudemos en afirmar que los estudios sobre las capacidades lingüisticas y de cognición de los póngidos contribuirán a esclarecerlo. En todo caso, en todos los casos, un poco más de modestia antropomórfica nos hará mucho bien en estas investigaciones.

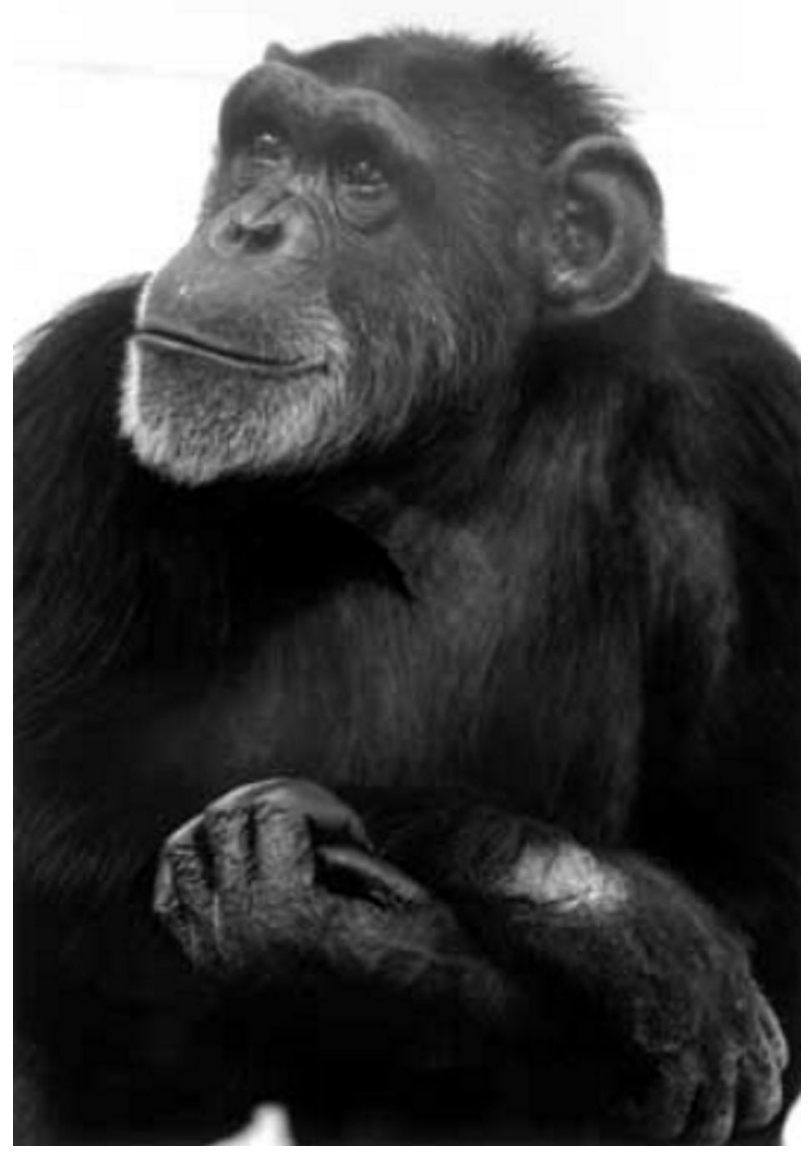

Washoe. Cortesía: friendsofwashoe.org 
$1 \mathrm{El}$ nombre proviene de pongo, un "monstruo" (el gorila probablemente) descrito por un marino inglés en el s. XVI. El gorila se descubre en 1847. Ahora se denomina póngidos a los grandes simios (Martínez-Contreras, 1996).

2 No analizaremos aquí la enseñanza del ASL a póngidos diferentes de los chimpancés. Recordemos solamente que Lyn Miles enseñó el ASL a un orangután y Francine Patterson a una gorila, Koko.

3 Washoe es el nombre de un pueblo al sur de Reno.

4 Entre 1947 y 1954, Keith y Catherine Hayes criaron a la chimpancé Viki junto con su propio hijo, tratando de enseñarle al simio a hablar como a un humano. Después de muchos años de entrenamiento, el animal sólo pronunciaba, en un "murmullo ronco", cuatro palabras.

5 La elección de los chimpancés no ha sido una elección fortuita. Su anatomía y su fisiología son muy cercanas de aquellas de los humanos. ¿No compartimos acaso casi el 98\% de nuestra información genética con ellos? Los chimpancés tienen una inteligencia general muy alta, y son capaces de desarrollar lazos afectivos con los hombres.

6 Así nombrado en honor de Robert M. Yerkes, pues Rumbaugh empezó sus actividades en el Yerkes Regional Primate Research Center de Atlanta.

7 Kanzi es el nombre del -ahora famoso- chimpancé macho Bonobo, es decir, de la especie grácil de los chimpancés, mal denominada Pan paniscus, pues el animal no es más pequeño que

Recibido: 20 de junio de 2009 Aceptado: 20 de diciembre de 2009
Gardner, Allen y Beatrix Gardner (1989a): Teaching Sign Language to Chimpanzees, Albany: State University of New York Press.

Gardner, Allen y Beatrix Gardner (1989b): "Prelinguistic Development of Children and Chimpanzee", Human EvoIution 4: 433-460.

Fouts, Roger (1979): "Strategies for Primate Language Training", en Schiefelbusch, Richard L. (ed.), Ape to Child, Bloomington: Indiana University Press, pp. 295-323.

Fouts, Roger (1989a): "Loulis in Conversation with Cross-Fostered Chimpanzees", en Gardner, R. Allen et al. (eds.), Teaching Sign Language to Chimpanzees, Albany: State University of New York Press, pp. 293307.

Fouts, Roger et al. (1989b): "The Infant Loulis Learns Signs from Cross-Fostered Chimpanzees", en Gardner, R. Allen et al. (eds.), Teaching Sign Language to Chimpanzees, Albany: State University of New York Press, pp. 280-292.

Fouts, Roger, Hirsch, Alan y Deborah H. Fouts (1982): "Cultural Transmission of a Human Language in a Chimpanzee Mother/Infant Relationship", en Fitzgerald, Hiram E., Mullins, J. A. y Gage, P. (eds.), Psychobiological Perspectives: Child Nurturance Series, vol. 111, New York: Plenum Press, pp. 159-193.

Martínez-Contreras, Jorge (1996): "The First Scientific Observations on Orangutan Behavior", Primate Report 45: 45-64.

Martínez-Contreras, Jorge (2002a): "Hombres y animales. Naturaleza compartida. Montaigne y Charron, pensadores contemporáneos", Ludus Vitalis 10(17): 37-50. 
Martínez-Contreras, Jorge (2002b): "Köhler y Yerkes: la búsqueda experimental de la inteligencia en primates no humanos", en Gutiérrez Lombardo, Raúl; Martinez-Contreras, Jorge y José Luis Vera Cortés (eds.), Naturaleza y diversidad humana. Estudios evolucionistas, México, D.F.: CEFPSVLT, pp. 185-205.

Martinez-Contreras, Jorge (2006): "Las primeras descripciones de antropoides en el Siglo XVII y su importancia para la filosofía de la evolución", en Rosas-López, Alejandro (ed.), Filosofía, Darwinismo y Evolución, Bogotá: Universidad Nacional, pp. 183-217.

Premack, David y G. Woodruff (1978): "Does the Chimpanzee have a Theory of Mind?", Behavioural and Brain Sciences 4: 515-526.

Premack, David y Ann James Premack (1983): The Mind of an Ape, New York: Norton.
Rumbaugh, Duane (1977): Language Learning by a Chimpanzee: the LANA project, New York: Academic Press.

Savage-Rumbaugh, E. Sue (1986): Ape Language: From Conditioned Response to Symbol, New York: Columbia University Press.

Savage-Rumbaugh, E. Sue y Lewin, Roger (1994): Kanzi: the Ape at the Brink of the Human Mind, New York: John Wiley \& Sons. 